\title{
Normal Supercharacter Theory
}

\author{
Farid Aliniaeifard $\|^{p}$ \\ ${ }^{1}$ York University, Toronto, Canada M3J 1P3
}

\begin{abstract}
There are three main constructions of supercharacter theories for a group $G$. The first, defined by Diaconis and Isaacs, comes from the action of a group $A$ via automorphisms on our given group $G$. Another general way to construct a supercharacter theory for $G$, defined by Diaconis and Isaacs, uses the action of a group $A$ of automorphisms of the cyclotomic field $Q\left[\zeta_{|G|}\right]$. The third, defined by Hendrickson, is combining a supercharacter theories of a normal subgroup $N$ of $G$ with a supercharacter theory of $G / N$. In this paper we construct a supercharacter theory from an arbitrary set of normal subgroups of $G$. We show that when we consider the set of all normal subgroups of $G$, the corresponding supercharacter theory is related to a partition of $G$ given by certain values on the central primitive idempotents. Also, we show the supercharacter theories that we construct can not be obtained via automorphisms or a single normal subgroup.

Résumé. Il y a trois constructions principales de théories des supercaractères pour un groupe $G$. La première, définie par Diaconis et Isaacs, provient de l'action d'un groupe $A$ par automorphismes sur un groupe donné $G$. Une autre méthode générale pour construire la théorie des supercaractères de $G$, définie par Diaconis et Isaacs, utilise l'action d'un groupe $A$ d'automorphismes du corps cyclotomique $Q\left[\zeta_{|G|}\right]$. La troisième, définie par Hendrickson, combine une théorie de supercaractères d'un sous-groupe distingué $N$ de $G$ avec une théorie des supercaractères de $G / N$. Dans cet article, nous construisons une théorie des supercaractères à partir d'un ensemble arbitraire de sous-groupes distingués de $G$. Nous montrons que lorsqu'on considère l'ensemble de tous les sous-groupes distingués de $G$, la théorie des supercarcatères correspondante est liée à une partition de $G$ donnée par certaines valeurs des idempotents centraux primitifs. Nous montrons aussi que les théories des supercaractères que nous construisons ne peuvent pas être obtenues par automorphismes ou par un unique sous-groupe distingué.
\end{abstract}

Keywords. Finite Group, Character Theory, Supercharacter Theory

\section{Introduction}

Let $G$ be a finite group, we denote the set of irreducible characters of $G$ by $\operatorname{Irr}(G)$. The conjugacy class containing $g$ and its cardinality are denoted by $C_{g}$ and $m_{g}$ respectively. For a subset $S$ of $G$, let $\widehat{S}=\sum_{s \in S} s$.

Let $U_{n}(q)$ denote the group of $n \times n$ unipotent upper triangular matrices over a finite field $\mathbb{F}_{q}$. The classification of the irreducible characters of $U_{n}(q)$ is a well-known wild problem, provably intractable for arbitrary $n$. In order to find a more tractable way to understand the representation theory of $U_{n}(q)$, André (1995) defines and constructs supercharacter theory. Yan (2001) shows how to replace André's

\footnotetext{
†Email: faridanfemathstat.yorku.ca. 
construction with more elementary methods. Diaconis and Isaacs (2008) axiomatize the concept of supercharacter theory for an arbitrary group. They mention how to obtain a supercharacter theory for $G$ from the action of $A$ on $G$ by automorphisms. They also generalize André's original construction to define a supercharacter theory for algebra groups, a group of the form $1+J$ where $J$ is a finite dimensional nilpotent associative algebra over a finite field $\mathbb{F}$ of characteristic $p$. Later, in Hendrickson (2012), it was shown that how to construct other supercharacter theories for an arbitrary group $G$ by combining certain supercharacter theory for a normal subgroup $N$ of $G$ with a supercharacter theory for $G / N$. Also in Aguiar et al. (2012) the authors obtain a relationship between the supercharacter theory of all unipotent upper triangular matrices over a finite field $\mathbb{F}_{q}$ simultaneously and the combinatorial Hopf algebra of symmetric functions in non-commuting variables.

Let $N(G)$ be the set of all normal subgroups of $G$. Let $S(G)$ be the set of all subsemigroups $A$ of $N(G)$ such that

1. $\{1\}, G \in A$.

2. $A$ is closed under intersection.

Define for $N \in A$

$$
N_{A}^{\circ}=N \backslash \bigcup_{K \in A, K<N} K .
$$

For simplicity of notation, we write $N^{\circ}$ instead of $N_{A}^{\circ}$ when it is clear that $N$ is in $A$. We will show that $\left\{N^{\circ}: N \in A\right\}$ is the set of superclasses of a supercharacter theory, and we call such supercharacter theory the normal supercharacter theory generated by $A$. In general this supercharacter theories can not be constructed by the previous supercharacter theory constructions. Remark that when we have a larger set of normal subgroups, the normal supercharacter theory we obtain will be finer. In particular the finest normal supercharacter theory is obtained when we consider the set of all normal subgroups of $G$, and is related to a partition of $G$ given by certain values on the central primitive idempotents.

In Section 2, we review definitions and notations for supercharacter theories. In particular we mention the known constructions of supercharacter theories. Next in Section 3, we define our normal supercharacter theory construction. We also show that the finest normal supercharacter theory is obtained by considering certain values of the central primitive idempotents. In Section 4 , we show that the normal supercharacter theory can not be obtained by the previous general constructions.

\section{Background}

We first mention the definition of supercharacter theory by Diaconis and Isaacs (2008).

A supercharacter theory of a finite group $G$ is a pair $(\mathcal{X}, \mathcal{K})$ where $\mathcal{X}$ is a partition of $\operatorname{Irr}(G)$ and $\mathcal{K}$ is a partition of $G$ such that:

(a) $|\mathcal{K}|=|\mathcal{X}|$,

(b) for $X \in \mathcal{X}$, the character $\chi_{X}$, a nonzero character whose irreducible constituents lie in $X$, is constant on the parts of $\mathcal{K}$ and

(c) the set $\{1\} \in \mathcal{K}$. 
We will refer to characters $\chi_{X}$ as the supercharacters and to the member of $\mathcal{K}$ as superclasses.

Every finite group has two trivial supercharacter theories: the usual irreducible character theory and the supercharacter theory $(\{\{\mathbf{1}\}, \operatorname{Irr}(G) \backslash\{\mathbf{1}\}\},\{\{1\}, G \backslash\{1\}\})$, where $\mathbf{1}$ is the principal character of $G$.

The concept of a Schur ring is defined by Schur (1933). Hendrickson (2012) shows that there is a bijection between the supercharacter theories of a group $G$ and Schur rings over $G$ contained in $Z(\mathbb{C}[G])$, the center of $\mathbb{C}[G]$.

Definition 2.1 Let $G$ be a finite group. A subring $S$ of the group algebra $\mathbb{C}[G]$ is called a Schur ring over $G$ if there is a set partition $\mathcal{K}$ of $G$ such that $\{1\} \in \mathcal{K}, S=\mathbb{C}$-span $\{\widehat{K}: K \in \mathcal{K}\}$, and $\left\{g^{-1}: g \in K\right\} \in \mathcal{K}$ for all $K \in \mathcal{K}$.

Proposition 2.2 (Hendrickson, 2012, Proposition 2.4) Let G be a finite group. Then there is a bijection

$\{$ Supercharacter theories $(\mathcal{X}, \mathcal{K})$ of $G\} \longleftrightarrow\{$ Schur rings over $G$ contained in $Z(\mathbb{C}[G])\}$

$$
(\mathcal{X}, \mathcal{K}) \quad \longmapsto \quad \mathbb{C} \text {-span }\{\widehat{K}: K \in \mathcal{K}\} .
$$

In the proof of surjectivity of the above bijection, Hendrickson does not need the condition $\left\{g^{-1}: g \in\right.$ $K\} \in \mathcal{K}$ from the definition of Schur ring. So we have the following corollary.

Corollary 2.3 Let $G$ be a finite group and let $\mathcal{K}$ be a partition of $G$. Then the following statements are equivalent.

1. $\mathcal{K}$ is the set of superclasses of a supercharacter theory.

2. $\{1\} \in \mathcal{K}$ and $\mathbb{C}$-span $\{\widehat{K}: K \in \mathcal{K}\}$ is a subring of $Z(\mathbb{C}[G])$.

Definition. A superclass theory is a partition $\mathcal{K}$ of $G$ satisfying one of the two equivalent conditions in Corollary 2.3 .

Now we discuss two main methods of constructing supercharacter theories of an arbitrary finite group.

\subsection{A Group Acts Via Automorphisms on a Given Group}

Given finite groups $A$ and $G$, we say that $A$ acts via automorphisms on $G$ if $A$ acts on $G$ as a set, and in addition $(g h) \cdot x=(g . x)(h . x)$ for all $g, h \in G$ and $x \in A$. An action via automorphisms of $A$ on $G$ determines and is determined by a homomorphism $\phi: A \rightarrow A u t(G)$.

Suppose that $A$ is a group that acts via automorphisms on our given group $G$. It is well known that $A$ permutes both the irreducible characters of $G$ and the conjugacy classes of $G$. By a lemma of R. Brauer, the permutation characters of $A$ corresponding to these two actions are identical, and so the numbers of $A$-orbits on $\operatorname{Irr}(G)$ and on the set of classes of $G$ are equal (See Theorem 6.32 and Corollary 6.33 of Isaacs (1994)). It is easy to see that these orbit decompositions yield a supercharacter theory $(\mathcal{X}, \mathcal{K})$ where members of $\mathcal{X}$ are the $A$-orbits on $\operatorname{Irr}(G)$ and members of $\mathcal{K}$ are the unions of the $A$-orbits on the classes of $G$. It is clear that in this situation, the sum of the characters in an orbit $X \in \mathcal{X}$ is constant on each member of $\mathcal{K}$. We denote by $\operatorname{AutSup}(G)$ the set of all such supercharacter theories of $G$. 


\subsection{Action of Automorphisms of The Cyclotomic Field $Q\left[\zeta_{|G|}\right]$}

Another general way to construct a supercharacter theory for $G$ uses the action of a group $A$ of automorphisms of the cyclotomic field $Q\left[\zeta_{|G|}\right]$, where $\zeta_{|G|}$ is a primitive $|G|$ th root of unity. Given $\sigma \in A$, there is a unique positive integer $r<|G|$ such that $\sigma\left(\zeta_{|G|}\right)=\zeta_{|G|}^{r}$, and we let $\sigma$ carry the class of $g \in G$ to the class of $g^{r}$. In this case too, we take $\mathcal{X}$ to be the set of $A$-orbits on $\operatorname{Irr}(G)$, and again, $\mathcal{K}$ is the set of unions of the various $A$-orbits on conjugacy classes. We denote by $\operatorname{ACSup}(G)$ the set containing above supercharacter theory.

\subsection{The $*$-Product}

Suppose that $A$ is a group that acts via automorphisms on our given group $G$. Let $\operatorname{Sup}(G)$ be the set of all supercharacter theories of $G$. We say that $(\mathcal{X}, \mathcal{K}) \in \operatorname{Sup}(G)$ is $A$-invariant if the action of $A$ fixes each part $K \in \mathcal{K}$ set-wise. We denote by $\operatorname{Sup}_{A}(G)$ the set of $A$-invariant supercharacter theories of $G$. Note that if $N$ is normal in $G$, then $\mathcal{C} \in \operatorname{Sup}(N)$ is $G$-invariant if and only if its superclasses are union of conjugacy classes of $G$. Also, if $M, N$ are normal subgroup of $G$ and $N<M$, then a supercharacter theory of $M / N$ is $G / N$-invariant if and only if it is $G$-invariant.

Notation. Let $N$ be a normal subgroup of a group $G$. If $\mathcal{L}$ is a set of subsets of $G / N$, then we define $\widetilde{\mathcal{L}}=\left\{\cup_{N g \in L} N g: L \in \mathcal{L}\right\}$. Let $\psi \in \operatorname{Irr}(N)$. We define $\operatorname{Irr}(G \mid \psi)=\left\{\chi \in \operatorname{Irr}(G):\left[\chi_{N}, \psi\right]>0\right\}$. If $\mathcal{Z}$ is a set of subsets of $\operatorname{Irr}(N)$, then we define $\mathcal{Z}^{G}=\left\{\cup_{\psi \in Z} \operatorname{Irr}(G \mid \psi): Z \in \mathcal{Z}\right\}$. Now consider $(\mathcal{X}, \mathcal{K}) \in \operatorname{Sup}_{G}(N)$. Since $\left\{1_{N}\right\} \in \mathcal{X}$, one part of $\mathcal{X}^{G}$ is

$$
\left\{1_{N}\right\}^{G}=\{\chi \in \operatorname{Irr}(G): N \subseteq \operatorname{ker} \chi\},
$$

which we identify with $\operatorname{Irr}(G / N)$ in the usual natural way.

Theorem 2.4 (Hendrickson, 2012, Theorem 4.3) Let $G$ be a group and $N$ be a normal subgroup of $G$. Let $\mathcal{C}=(\mathcal{X}, \mathcal{K}) \in \operatorname{Sup}_{G}(N)$ and $\mathcal{D}=(\mathcal{Y}, \mathcal{L}) \in \operatorname{Sup}(G / N)$. Then

$$
\left(\mathcal{Y} \cup \mathcal{X}^{G} \backslash\{\operatorname{Irr}(G / N)\}, \mathcal{K} \cup \widetilde{\mathcal{L}} \backslash\{N\}\right)
$$

is a supercharacter theory of $G$.

We call the supercharacter theory of $G$ constructed in the preceding theorem the $*$-product of $(\mathcal{X}, \mathcal{K})$ and $(\mathcal{Y}, \mathcal{L})$, and write it as $(\mathcal{X}, \mathcal{K}) *(\mathcal{Y}, \mathcal{L})$. Also, let $\operatorname{Sup}^{*}(G)$ denote the set of all supercharacter theories of $G$ obtained by *-product.

\section{Normal Supercharacter Theory}

In this section we construct a supercharacter theory from an arbitrary set of normal subgroups. We call such supercharacter theory a normal supercharacter theory.

\subsection{Supercharacter Theory From Central Idempotents}

In this subsection, we consider a partition of conjugacy classes and irreducible characters given by certain values of central primitive idempotent. In the next subsection, we will see that this is a supercharacter theory and is given by the finest normal supercharacter theory. 
By (Lam, 1991, Proposition 8.15) every character $\chi \in \operatorname{Irr}(G)$ has a corresponding central primitive idempotent

$$
e_{\chi}=|G|^{-1} \chi(1) \sum_{g \in G} \chi\left(g^{-1}\right) g
$$

These idempotents are orthogonal, i.e, $e_{\chi} e_{\phi}=0$ when $\chi \neq \phi$. Recall that

$$
\widehat{C_{g}}=\sum_{i} m_{g} \chi_{i}(1)^{-1} \chi_{i}(g) e_{\chi_{i}}
$$

Therefore,

$$
\begin{gathered}
m_{g} 1-\widehat{C_{g}}=m_{g} 1-\sum_{i} m_{g} \chi_{i}(1)^{-1} \chi_{i}(g) e_{\chi_{i}}=m_{g}\left(1-\sum_{i} \chi_{i}(1)^{-1} \chi_{i}(g) e_{\chi_{i}}\right)= \\
m_{g}\left(\sum_{i} e_{\chi_{i}}-\sum_{i} \chi_{i}(1)^{-1} \chi_{i}(g) e_{\chi_{i}}\right)=m_{g}\left(\sum_{i}\left(1-\chi_{i}(1)^{-1} \chi_{i}(g)\right) e_{\chi_{i}}\right) \\
\Rightarrow 1-\frac{\widehat{C_{g}}}{m_{g}}=\sum_{i}\left(1-\frac{\chi_{i}(g)}{\chi_{i}(1)}\right) e_{\chi_{i}}=\sum_{i}\left(1-\frac{\chi_{i}(g)}{\chi_{i}(1)}\right) e_{\chi_{i}} .
\end{gathered}
$$

\begin{tabular}{|c|c|c|c|c|c|c|c|}
\hline Classes & (1) & (1 2) & $\left(\begin{array}{lll}1 & 2 & 3\end{array}\right)$ & $\left(\begin{array}{llll}1 & 2 & 3 & 4\end{array}\right)$ & $(12345)$ & $(12)(34)$ & $(12)(345)$ \\
\hline$\chi_{1}$ & 1 & 1 & 1 & 1 & 1 & 1 & 1 \\
\hline$\chi_{2}$ & 1 & -1 & 1 & -1 & 1 & 1 & -1 \\
\hline$\chi_{3}$ & 4 & 2 & 1 & 0 & -1 & 0 & -1 \\
\hline$\chi_{4}$ & 4 & -2 & 1 & 0 & -1 & 0 & 1 \\
\hline$\chi_{5}$ & 5 & -1 & -1 & 1 & 0 & 1 & -1 \\
\hline$\chi_{6}$ & 5 & 1 & -1 & -1 & 0 & 1 & 1 \\
\hline$\chi_{7}$ & 6 & 0 & 0 & 0 & 1 & -2 & 0 \\
\hline
\end{tabular}

Look at the last equation i.e., $1-\frac{\widehat{C_{g}}}{m_{g}}=\sum_{i}\left(1-\frac{\chi_{i}(g)}{\chi_{i}(1)}\right) e_{\chi_{i}}$. Let

$$
E_{g}=\left\{e_{\chi_{i}}: 1-\frac{\chi_{i}(g)}{\chi_{i}(1)} \neq 0\right\}, K_{g}=\cup_{E_{g}=E_{h}} C_{h} \text {, and } U_{g}=\cup_{E_{h} \subseteq E_{g}} C_{h} .
$$

In following example we will see that $\left\{K_{g}: g \in G\right\}$ is a superclass theory.

Example 3.1 The character table of $S_{5}$ is

By definition

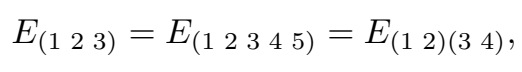

then

$$
K_{(123)}=K_{(12345)}=K_{(12)(34)} .
$$

Also, 


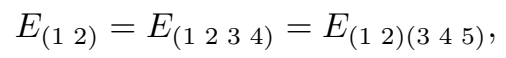

then

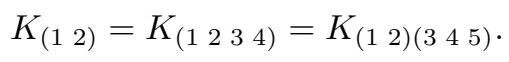

One can check that $\left(\left\{\{1\}, K_{(123)}, K_{(12)}\right\},\left\{\left\{\chi_{1}\right\},\left\{\chi_{2}\right\},\left\{\chi_{3}, \chi_{4}, \chi_{5}, \chi_{6}, \chi_{7}\right\}\right\}\right)$ is a supercharacter theory.

In the above example $\left\{K_{g}: g \in G\right\}$ forms a superclass theory. A natural question arises: does $\left\{K_{g}: g \in G\right\}$ always give rise to a superclass theory? We will answer this question in Corollary 3.7 .

\subsection{Normal Supercharacter Theory}

In this subsection we construct our normal supercharacter theory. We will show the finest normal supercharacter theory is related to $\left\{K_{g}: g \in G\right\}$ the partition of $G$ given by $\left\{E_{g}: g \in G\right\}$ a subset of the set of all subsets of central primitive idempotents. We need the following definitions and notations in the sequel.

If $(P, \leq)$ is a poset and $\mathbb{C}^{P \times P}$ is the set of all functions $\alpha: P \times P \rightarrow \mathbb{C}$, the associated incidence algebra is

$$
A(P)=\left\{\alpha \in \mathbb{C}^{P \times P}: \alpha(s, u)=0 \text { unless } s \leq u\right\} .
$$

The Mobius function $\mu \in A(P)$ is defined recursively by the following rules:

$$
\mu(s, s)=1
$$

and

$$
\mu(s, u)=-\sum_{s \leq t<u} \mu(t, u), \text { for all } s<u \text { in } P .
$$

It is immediate from this definition that

$$
\sum_{s \leq t \leq u} \mu(t, u)= \begin{cases}1 & \text { if } s=u \\ 0 & \text { otherwise }\end{cases}
$$

Let $N(G)$ be the set of all normal subgroup of $G$. Note that the product of two normal subgroup is a normal subgroup. We can see that $N(G)$ is a semigroup. Recall that $S(G)$ is the set of all subsemigroups $A$ of $N(G)$ such that

1. $\{1\}, G \in A$.

2. $A$ is closed under intersection.

Note that every element $N \in A$ is a normal subgroup of $G$. We define for an element $N \in A$

$$
N_{A}^{\circ}=N \backslash \bigcup_{K \in A, K<N} K
$$


For simplicity of notation, we write $N^{\circ}$ instead of $N_{A}^{\circ}$ when it is clear that $N$ is in $A$. Note that

$$
\widehat{N}=\sum_{H \in A,\{1\} \leq H \leq N} \widehat{H}^{\circ}
$$

Thus, by Mobius Inversion Theorem we have

$$
\widehat{N^{\circ}}=\sum_{H \in A,\{1\} \leq H \leq N} \mu(H, N) \widehat{H} .
$$

Example 3.2 Let $G=C_{2} \times C_{4}=\langle a\rangle \times\langle b\rangle$. Here is the Hasse diagram for $N(G)$, and $\mu(H, G)$ for every $H \in N(G)$ is written above the vertex $H$.

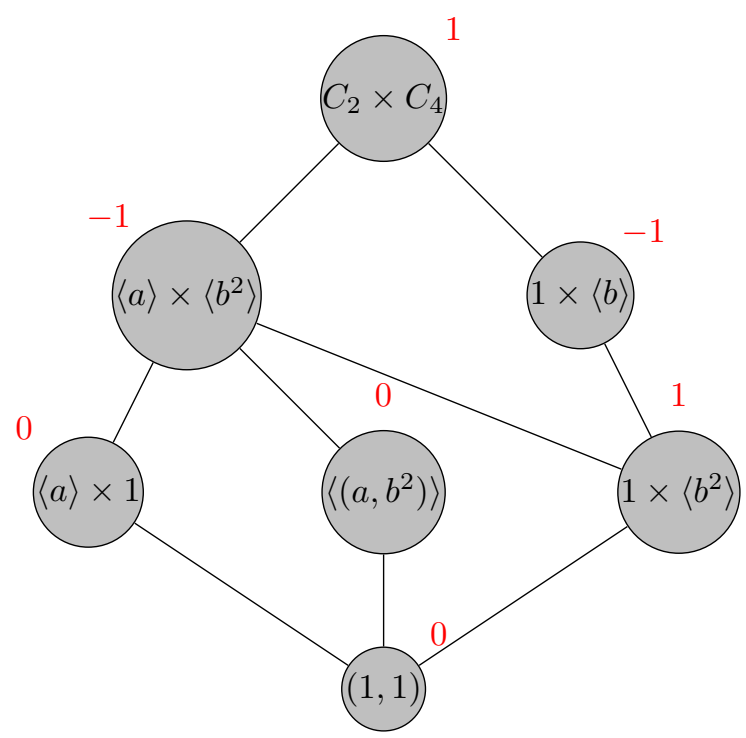

By the diagram above it is easy to see that $\widehat{C}_{2} \times C_{4}{ }^{\circ}=\sum_{\{(1,1)\} \leq H \leq C_{2} \times C_{4}} \mu\left(H, C_{2} \times C_{4}\right) \hat{H}$.

Theorem 3.3 Let $A \in S(G)$. Then $\mathcal{K}=\left\{N^{\circ} \neq \emptyset: N \in A\right\}$ is a superclass theory.

Proof. Let $N^{\circ}, H^{\circ} \in \mathcal{K}$. Then by the definition of $N^{\circ}$ and $H^{\circ}$, it is easy to see that $N^{\circ}=H^{\circ}$ or $N^{\circ} \cap H^{\circ}=\emptyset$. Furthermore, $\bigcup_{N \in A} N^{\circ}=G$. Therefore, $\mathcal{K}$ is a partition of $G$ such that $\{1\} \in \mathcal{K}$.

Let $g \in N^{\circ}$. Then $C_{g} \in N$. If $C_{g} \cap H \neq \emptyset$ for some normal subgroup $H$ in $A$ and $H \subset N$, then $g \in C_{g} \subseteq H$. Thus, $g \notin N^{\circ}$, yielding a contradiction. Therefore, we must have $C_{g} \subseteq N^{\circ}$. So every member of $\mathcal{K}$ is a union of conjugacy classes of $G$. We have

$$
\widehat{N^{\circ}}=\sum_{H \in A, H \leq N} \mu(H, N) \widehat{H} \text { and } \widehat{N}=\sum_{H \in A, H \leq N} \widehat{H^{\circ}} .
$$

Therefore, $\mathbb{C}-\operatorname{span}\left\{\widehat{N^{\circ}}: N \in A\right\}=\mathbb{C}-\operatorname{span}\{\widehat{N}: N \in A\}$. Since $A$ is closed under product, $\mathbb{C}$ $\operatorname{span}\{\widehat{N}: N \in A\}$ is a subalgebra of $Z(\mathbb{C}[G])$. We conclude by Corollary 2.3 that $\mathcal{K}$ is a superclass 
theory.

As we see in Theorem 3.3 for every $A \in S(G),\left\{N^{\circ}: N \in A\right\}$ is a superclass theory. We say a supercharacter theory $(\mathcal{X}, \mathcal{K})$ is a normal supercharacter theory if $\mathcal{K}=\left\{N^{\circ}: N \in A\right\}$ for some $A \in S(G)$. We denote by $N \operatorname{Sup}(G)$ the set of all possible normal supercharacter theories of $G$.

A subgroup of $G$ is normal if and only if it is the union of a set of conjugacy classes of $G$. We have an equivalent characterization of normality in terms of the kernels of irreducible characters. Recall that the kernel of a character $\chi$ of $G$ is the set $\operatorname{ker} \chi=\{g \in G: \chi(g)=\chi(1)\}$. This is just the kernel of any representation whose character is $\chi$, and so $\operatorname{ker} \chi$ is normal subgroup. A subgroup of $G$ is normal if and only if it is the intersection of the kernels of some finite set of irreducible characters (James and Liebeck. 1993. Proposition 17.5); thus the normal subgroups of $G$ are the subgroups which we can construct from the character table of $G$.

Recall that

$$
E_{g}=\left\{e_{\chi_{i}}: 1-\frac{\chi_{i}(g)}{\chi_{i}(1)} \neq 0\right\} \text { and } K_{g}=\cup_{E_{g}=E_{h}} C_{h} .
$$

Consider $N(G) \in S(G)$. We show that $K_{g}=N^{\circ}$ for a normal subgroup of $G$, and if for a normal subgroup $N$ of $G, N^{\circ} \neq \emptyset$, then there is a $g \in N$ such that $K_{g}=N^{\circ}$. First we prove the following lemma.

Lemma 3.4 Assume that $E_{g}=\left\{e_{\chi_{i}}: i \in I\right\}$. If

$$
N=\bigcap_{\chi \in \operatorname{Irr}(G) \backslash\left\{\chi_{i}: i \in I\right\}} \operatorname{ker} \chi,
$$

then $K_{g}=N^{\circ}$.

Proof. Let $k \in K_{g}$. Then $E_{k}=E_{g}$, and so $k \in \operatorname{ker} \chi$ for every $\chi \in \operatorname{Irr}(G) \backslash\left\{\chi_{i}: i \in I\right\}$. Therefore, $k \in N$. Let $H$ be a normal subgroup of $G$ such that $H \subset N$. Then at least there is an irreducible character $\psi \in \operatorname{Irr}(G)$ such that $H \subseteq \operatorname{ker} \psi$, but $N \nsubseteq \operatorname{ker} \psi$. If $k \in H$, then

$$
k \in \bigcap_{\chi \in \operatorname{Irr}(G) \backslash\left\{\chi_{i}: i \in I\right\}} \operatorname{ker} \chi \cap \operatorname{ker} \psi,
$$

and so $E_{k} \neq E_{g}$. Thus, $k \notin K_{g}$, yielding a contradiction. Therefore, $k$ is in $N$, but $k$ is not in any normal subgroup $H$ of $G$ such that $H \subset N$, i.e., $k \in N^{\circ}$. Therefore, $K_{g} \subseteq N^{\circ}$.

Let $h \in N^{\circ}$. Then $E_{h} \subseteq E_{g}$. If $E_{g} \neq E_{h}$, there is an irreducible character $\psi \in\left\{\chi_{i}: i \in I\right\}$ such that $h \in \operatorname{ker} \psi$. Let $H=N \cap \operatorname{ker} \psi$. Then $h \notin N \backslash(N \cap \operatorname{ker} \psi)$. Therefore, $h \notin N^{\circ}$, yielding a contradiction. We can conclude that $E_{g}=E_{h}$, and so $h \in K_{g}$. Thus, $N^{\circ} \subseteq K_{g}$.

Theorem 3.5 Let $G$ be a group.

1. For every $g \in G$, there is a normal subgroup $N$ of $G$ such that $K_{g}=N^{\circ}$.

2. Let $N$ be a normal subgroup of $G$. If $N^{\circ} \neq \emptyset$, then for every $g \in N^{\circ}, K_{g}=N^{\circ}$. 
Proof. (1) Let $E_{g}=\left\{e_{\chi_{i}}: i \in I\right\}$ and let

$$
N=\bigcap_{\chi \in \operatorname{Irr}(G) \backslash\left\{\chi_{i}: i \in I\right\}} \operatorname{ker} \chi .
$$

Then by Lemma 3.4, $K_{g}=N^{\circ}$.

(2) Let $N$ be a normal subgroup of $G$ such that $N^{\circ} \neq \emptyset$. Let $g \in N^{\circ}$. We show that $K_{g}=N^{\circ}$. Assume that $N=\bigcap_{i \in I} \operatorname{ker} \chi_{i}$. If there is an irreducible character $\chi \in \operatorname{Irr}(G) \backslash\left\{\chi_{i}: i \in I\right\}$ such that $g \in \operatorname{ker} \chi$, then $g \in H=\bigcap_{i \in I}$ ker $\chi_{i} \cap$ ker $\chi$. Thus, $g \in H \subset N$, and so $g \notin N^{\circ}$, yielding a contradiction. Therefore, $E_{g}=\left\{e_{\chi}: \chi \in \operatorname{Irr}(G) \backslash\left\{\chi_{i}: i \in I\right\}\right\}$. By Lemma 3.4 $K_{g}=N^{\circ}$.

Corollary 3.6 Let $G$ be a group. Then for every $g \in G, U_{g}$ is a normal subgroup of $G$.

Proof. Recall that

$$
U_{g}=\bigcup_{E_{h} \subseteq E_{g}} C_{h}
$$

Let $E_{g}=\left\{e_{\chi_{i}}: i \in I\right\}$. We show that

$$
U_{g}=N=\bigcap_{\chi \in \operatorname{Irr}(G) \backslash\left\{\chi_{i}: i \in I\right\}} \operatorname{ker} \chi .
$$

Let $h \in N$. We have $h \in \operatorname{ker} \chi$ for every $\chi \in \operatorname{Irr}(G) \backslash\left\{\chi_{i}: i \in I\right\}$. Therefore, $E_{h} \subseteq E_{g}$, and so $h \in U_{g}$. We conclude that $N \subseteq U_{g}$.

Let $h \in U_{g}$. Then $E_{h} \subseteq E_{g}$, and so $h \in \bigcap_{\chi \in \operatorname{Irr}(G) \backslash\left\{\chi_{i}: i \in I\right\}} \operatorname{ker} \chi=N$. Therefore, $U_{g} \subseteq N$.

As we mentioned before, the finest normal supercharacter theory is obtained when we generate a normal supercharacter theory by $N(G)$ the set of all normal subgroups of $G$. In the following corollary we show that the finest normal supercharacter theory is equal to the supercharacter theory with $\left\{K_{g}: g \in G\right\}$ as the set of superclasses. And since every $K_{g}$ is related to a set of central primitive idempotents, we can see that the finest normal supercharacter theory corresponds to a set of subsets of central primitive idempotents.

Corollary 3.7 Let $G$ be a group. Then $\left\{K_{g}: g \in G\right\}=\left\{N^{\circ}: N \in N(G)\right\}$ is the finest normal superclass theory.

Proof. The normal supercharacter theory generated by $N(G)$ has $\left\{N^{\circ}: N \in N(G)\right\}$ as the set of superclasses. Since every non-empty $N^{\circ}$ is equal to $K_{g}$ for some $g \in G$. We have $\left\{N^{\circ}: N \in N(G)\right\}=$ $\left\{K_{g}: g \in G\right\}$. Therefore, $\left\{K_{g}: g \in G\right\}$ is a superclass theory.

\section{$4 N \operatorname{Sup}(G)$ is not a subset of the union of $\operatorname{AutSup}(G), A C S u p(G)$, and $\operatorname{Sup}^{*}(G)$}

In the following example we show that $\operatorname{Sup}^{*}(G) \cap \operatorname{AutSup}(G)$ is not a subset of $N \operatorname{Sup}(G)$ and there is a normal supercharacter theory which is not in the union of $\operatorname{AutSup}(G), \operatorname{ACSup}(G)$, and $\operatorname{Sup}^{*}(G)$.

Example. Let $G=C_{3} \times C_{4}$. Note that the supercharacter theory correspond to superclass theory $\left\{C_{g}: g \in G\right\}$ is in $\operatorname{Sup}^{*}(G) \cap \operatorname{AutSup}(G)$, but it is not in $N \operatorname{Sup}(G)$. Therefore, $\operatorname{Sup}^{*}(G) \cap \operatorname{AutSup}(G)$ 
is not a subset of $N \operatorname{Sup}(G)$. We now construct the normal supercharacter theory generated by $A$, where $A$ is the smallest semigroup in $S(G)$ containing $\left\{C_{3} \times 1,1 \times C_{4}\right\}$ and we show that this supercharacter theory is not in the union of $\operatorname{AutSup}(G), \operatorname{ACSup}(G)$, and $\operatorname{Sup}^{*}(G)$.

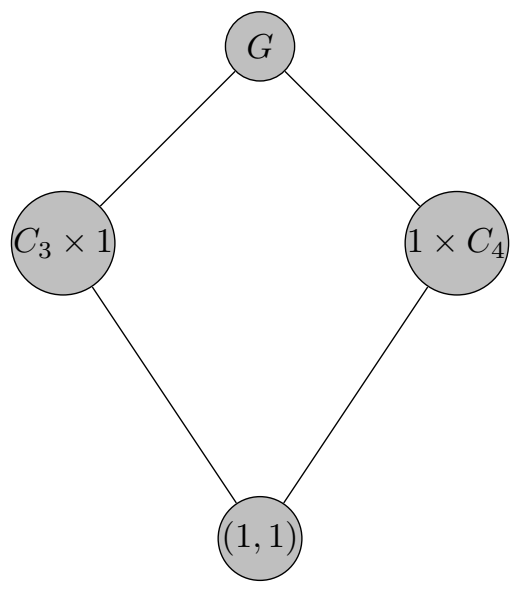

The set of superclasses for the normal supercharacter theory generated by $A$ is

$$
\left\{(1,1),\left\{(g, 1),\left(g^{2}, 1\right)\right\},\left\{(1, h),\left(1, h^{2}\right),\left(1, h^{3}\right)\right\},\left\{(g, h),\left(g, h^{2}\right),\left(g, h^{3}\right),\left(g^{2}, h\right),\left(g^{2}, h^{2}\right),\left(g^{2}, h^{3}\right)\right\}\right\} .
$$

Let $(\mathcal{X}, \mathcal{K}) \in \operatorname{AutSup}(G)$. Since $A u t(G) \cong \mathbb{Z}_{2} \times \mathbb{Z}_{2}$, every orbit has at most 4 members. Note that the members of $\mathcal{K}$ are the unions of the $A$-orbits on the classes of $G$. Therefore, every members of $\mathcal{K}$ has at most cardinality 4 . But we have a superclass with cardinality 6 in the normal supercharacter theory generated by $A$. Thus, this normal supercharacter theory for $G$ is not in $\operatorname{Aut} S u p(G)$.

Since $\left|A u t\left(Q\left[\zeta_{|G|}: Q\right]\right)\right|=4$, the largest superclass in $\mathcal{K} \in A C S u p(G)$ has cardinality 4 . Note that the set of superclasses for the normal supercharacter theory generated by $A$ has a superclass of cardinality 6. Therefore, the normal supercharacter theory generated by $A$ is not in $A C \operatorname{Sup}(G)$.

Now we show that normal supercharacter theory generated by $A$ is not in $\operatorname{Sup}^{*}(G)$. If we choose a subgroup of order 2 and construct the $*$-product, then there is two superclass with cardinality 1 , but we only have one superclass with cardinality 1 in the normal supercharacter theory generated by $A$. Let us choose a subgroup of order 4 . Then $\left\{(g, 1),\left(g^{2}, 1\right)\right\}$ is not a superclass of this supercharacter theory. Now we choose a subgroup of order 3 , and construct the supercharacter theory by $*$-product. Then $\left\{(1, h),\left(1, h^{2}\right),\left(1, h^{3}\right)\right\}$ is not a superclass of this supercharacter theory.

Therefore, the normal supercharacter theory generated by $A$ is not in $\operatorname{AutSup}(G) \cup \operatorname{ACSup}(G) \cup$ $\operatorname{Sup}^{*}(G)$.

\section{Acknowledgements}

I wish to express my appreciation to my supervisor professor Bergeron who carefully read an earlier version of this paper and made significant suggestions for improvement. Also, I would like to thank 
Martin Isaacs, Nat Thiem, and Shu Xiao Li for their helpful comments. In the end, I wish to thank the referees who made useful suggestions.

\section{References}

M. Aguiar, C. André, C. Benedetti, N. Bergeron, Z. Chen, P. Diaconis, A. Hendrickson, S. Hsiao, M. Isaacs, A. Jedwab, K. Johnson, G. Karaali, A. Lauve, T. Le, S. Lewis, H. Li, K. Magaard, E. Marberg, J.-C. Novelli, A. Pang, F. Saliola, L. Tevlin, J.-Y. Thibon, N. Thiem, V. Venkateswaran, C. Vinroot, N. Yan, and M. Zabrocki. Supercharacters, symmetric functions in noncommuting variables, and related hopf algebras. Adv. Math., 229(4), 2012.

C. André. Basic characters of the unitriangular group. J. Algebra, 175, 1995.

C. André. Irreducible characters of finite algebra groups, in: Matrices and group representations. in: Textos Mat. Sér. B, 19, 1998.

C. André. The basic character table of the unitriangular group. J. Algebra, 241, 2001.

C. André. Basic characters of the unitriangular group (for arbitrary primes). Proc. Amer. Math. Soc., 130, 2002.

P. Diaconis and M. Isaacs. Supercharacters and superclasses for algebra groups. Trans. Amer. Math. Soc., 360, 2008.

A. O. F. Hendrickson. Supercharacter theory constructions corresponding to schur ring products. Comm. Algerba, 40, 2012.

I. M. Isaacs. Character theory of finite groups. Dover, New York, 1994.

G. James and M. Liebeck. Representations and Characters of Groups. Cambridge Univ. Press, Cambridge, UK, 1993.

T. Y. Lam. The first course in noncommutative rings. Springer-Verlag, New York, 1991.

I. Schur. Zur theorie der einfach transitiven permutationsgruppen. Sitzungsber. Preuss. Akad. Wiss. Phys.Math. Kl., 1933.

N. Yan. Representation theory of the finite unipotent linear groups. Unpublished Ph.D. Thesis, Department of Mathematics, University of Pennsylvania, 2001. 
